\title{
Observed Mass Profiles in Massive ETGs and Clusters and Implications for $\Lambda \mathrm{CDM}$
}

\author{
David A. Buote \\ Department of Physics and Astronomy, 4129 Frederick Reines Hall, \\ University of California, Irvine, Irvine, CA, 92697-4575, USA \\ email: buote@uci.edu
}

\begin{abstract}
In my talk I reviewed observations of the dark matter and total mass profiles in massive ETGs and clusters and their implications for $\Lambda$ CDM. In this brief proceedings, I have extracted just a subset of material from my talk due to space limitations.
\end{abstract}

Keywords. Dark Matter, Elliptical Galaxies, Galaxy Clusters

\section{Dark Matter Profiles}

For massive early-type galaxies (ETGs) the baryonic mass exceeds the dark matter (DM) within the stellar half-light radius $\left(R_{e}\right.$; e.g., Auger et al. 2010). It is therefore difficult to disentangle the DM profile from the stellar component, regardless of the observational tracer or technique employed. Typically profiles with either a central cusp or core are allowed by the observations so that, in particular, the NFW profile is consistent with, but not required by, the data. To distinguish between different model profiles, it is necessary to examine more DM-dominated systems. This means either going down in mass scale to the dwarfs or up in mass scale to galaxy clusters.

The typical result from X-ray observations of galaxy clusters is that the NFW model describes the DM profile very well (e.g., Pointecouteau et al. 2005). Unlike ETGs, clusters clearly disfavor a pseudo-isothermal DM profile with a core. With only a few exceptions (see below) the X-ray studies do not provide evidence for more complicated profiles than NFW (e.g., Einasto).

Gravitational lensing studies of clusters generally agree with the X-ray results. Very recent results from the CLASH survey (Umetsu et al. 2015) find that the mean surface density profile of the clusters in their sample is described well by the NFW profile while a cored-isothermal model is clearly disfavored. They also show that the Einasto model and other more complicated models fit the data comparably to NFW.

Beginning with the work by Sand and colleagues (e.g., Sand et al. 2002, 2004) using a combination of stellar dynamics (SD) and lensing, it was found that in some clusters the DM profile within essentially $R_{e}$ of the BCG is significantly shallower than NFW, indicating a radial logarithmic density slope of about -0.5 very similar to what is found in dwarf galaxies. Shallow central DM profiles have also been found in a few clusters studied in X-rays.

Moreover, some studies emphasized that, while the DM profile may be shallower than NFW, the NFW model fits the total mass profile quite well. This was originally pointed out for a couple of clusters in X-ray studies (e.g., Lewis et al. 2003; Zappacosta et al. 2006), but Newman and colleagues (Newman et al. 2013) have more recently highlighted this issue and examined its implications in detail. These results imply that dissipative processes are not very important for massive cluster formation. The mass scale where the dissipative processes becomes important can be identified when an NFW component 
distinct from the BCG is shown by the observations. In a recent paper, Newman et al. (2015) propose $10^{14} M_{\odot}$ for this transition based on a study of 10 systems. It is interesting that the most massive clusters (of which I am aware) from X-ray studies clearly indicating a distinct NFW DM component are RXJ 1159+5531 (Gastaldello et al. 2007; Humphrey et al. 2012) and RXJ 2315.7-0222 (Démoclès et al. 2010), which have virial masses very close to $10^{14} M_{\odot}$.

\section{Concentration-Mass Relation}

The deviation of self-similarity of $\Lambda \mathrm{CDM}$ profiles is quantified by the concentrationmass $(c-m)$ relation. The deviation is indeed small, since the concentration varies by only a factor of a few over about five decades in mass. There is significant intrinsic scatter in this relation that is essentially independent of mass. The slope and the scatter are not very sensitive to cosmological parameters, in particular $\sigma_{8}, \Omega_{m}$, and $w$.

To date there have been many X-ray studies of the $c-m$ relation for clusters, but since the launch of Chandra only one study has considered the mass range spanning massive ETGs and clusters. Buote et al. (2007) analyzed 39 low- $z$ systems spanning three decades in mass obtaining strong constraints on a power-law fit of the $c-m$ relation: slope $-0.172 \pm 0.026$ and normalization $9.0 \pm 0.4\left(\right.$ at $\left.10^{14} h^{-1} M_{\odot}\right)$. These values broadly agree with the Planck $\Lambda$ CDM values quoted by Dutton \& Macciò (2014), but they are marginally discrepant: X-ray slope is $\sim 3 \sigma$ higher, normalization slightly exceeds the $1 \sigma$ intrinsic scatter, and the X-ray intrinsic scatter of 0.10 is $\sim 2 \sigma$ lower than the $\Lambda$ CDM value of 0.11 .

Are these genuine discrepancies? Rasia et al. (2013) studied the $c-m$ relation inferred from X-ray cluster observations using hydrodynamical simulations. They found that the slope of the $c-m$ relation is not very sensitive to the ingredients of the simulations and to most effects in the X-ray observations and obtained good agreement with that of Buote et al. (2007). The normalization is more affected, and the simulations with cooling and star formation agree better with the observations, particularly once issues related to the fitting range of the halos are addressed consistently between the observations and simulations.

\section{References}

Auger, M. W., Treu, T., Bolton, A. S., et al. 2010, ApJ, 724, 511

Buote, D. A., Gastaldello, F., Humphrey, P. J., et al. 2007, ApJ, 664, 123

Démoclès, J., Pratt, G. W., Pierini, D., et al. 2010, A\&A, 517, A52

Dutton, A. A. \& Macciò, A. V. 2014, MNRAS, 441, 3359

Gastaldello, F., Buote, D. A., Humphrey, P. J., et al. 2007, ApJ, 669, 158

Humphrey, P. J., Buote, D. A., Brighenti, F., et al. 2012, ApJ, 748, 11

Lewis, A. D., Buote, D. A., \& Stocke, J. T. 2003, ApJ, 586, 135

Newman, A. B., Ellis, R. S., \& Treu, T. 2015, ArXiv e-prints, arXiv:1503.05282

Newman, A. B., Treu, T., Ellis, R. S., et al. 2013, ApJ, 765, 24

Pointecouteau, E., Arnaud, M., \& Pratt, G. W. 2005, A\& A, 435, 1

Rasia, E., Borgani, S., Ettori, S., Mazzotta, P., \& Meneghetti, M. 2013, ApJ, 776, 39

Sand, D. J., Treu, T., \& Ellis, R. S. 2002, ApJ, 574, L129

Sand, D. J., Treu, T., Smith, G. P., \& Ellis, R. S. 2004, ApJ, 604, 88

Umetsu, K., Zitrin, A., Gruen, D., et al. 2015, ArXiv e-prints, arXiv:1507.04385

Zappacosta, L., Buote, D. A., Gastaldello, F., et al. 2006, ApJ, 650, 777 